\title{
Preservation of cerebrospinal fluid cells to investigate intrathecal immunity in neurodegeneration
}

Olivia Levanthal

Stanford University

Tony Wyss-Coray

Stanford University

David Gate ( $\nabla$ dgate@stanford.edu )

Stanford University https://orcid.org/0000-0003-0481-9657

\section{Methodology}

Keywords: cerebrospinal fluid cells, intrathecal cells, neurodegeneration, adaptive immunity, innate immunity, T cells

Posted Date: April 28th, 2020

DOl: https://doi.org/10.21203/rs.3.rs-23747/v1

License: (c) (i) This work is licensed under a Creative Commons Attribution 4.0 International License. Read Full License 


\title{
Preservation of cerebrospinal fluid cells to investigate intrathecal immunity in neurodegeneration
}

\author{
Olivia Leventhal ${ }^{1}$, Tony Wyss-Coray ${ }^{1,2}$ and David Gate ${ }^{1,2, *}$ \\ ${ }^{1}$ Department of Neurology and Neurological Sciences, Stanford University School of Medicine, Stanford, \\ California, USA \\ ${ }^{2}$ Veterans Administration Palo Alto Healthcare system, Palo Alto, CA, USA \\ *Corresponding author: dgate@,stanford.edu
}

\section{AUTHORS' EMAIL ADDRESSES}

Olivia Leventhal: olivialeventhal@stanford.edu

Tony Wyss-Coray: twc@stanford.edu

David Gate: dgate@stanford.edu

\begin{abstract}
Background: Cerebrospinal fluid (CSF) provides basic mechanical and immunological protection to the brain. Historically, analysis of CSF has focused on protein changes, yet recent studies of neurodegenerative diseases have shed light on cellular alterations. Due to low cell numbers in the CSF, only recently have advances in molecular biology techniques allowed for the investigation of disease-related cellular changes. Chief among these advances is single cell RNA sequencing (scRNAseq). However, concerns such as batch effects and time constraints call for a standardized method for long-term preservation of CSF immune cells.

Results: We present a method for long-term cryopreservation of CSF immune cells for downstream scRNAseq analysis. Cells can be analyzed up to two years following CSF collection. On average, 4,961 live cells can be recovered from $10 \mathrm{~mL}$ CSF after cryopreservation, with no association between length of storage and cell viability. We demonstrate that scRNAseq detects $\mathrm{CD}^{+}$and $\mathrm{CD}^{+} \mathrm{T}$ cells, natural killer cells, plasma cells, B cells, and innate immune cells (monocytes and dendritic cells) within the CSF of 24 subjects.

Conclusions: The ability to store CSF cells long-term will enable researchers actively collecting CSF to investigate intrathecal immunity. This method will aid in the understanding of cellular and molecular changes to CSF immunity in neurodegenerative diseases.
\end{abstract}

Keywords: cerebrospinal fluid cells, intrathecal cells, neurodegeneration, adaptive immunity, innate immunity, T cells 


\section{BACKGROUND}

The cerebrospinal fluid (CSF) provides insight into brain physiology of living individuals. Biochemical analysis of CSF is routinely utilized as a diagnostic tool in neurodegeneration (1,2). For example, in Alzheimer's disease (AD), changes in protein levels of total tau, phosphorylated tau and amyloid- $\beta$ are indicative of disease pathology. However, while CSF biomarkers guide diagnosis of patients with neurodegenerative diseases, the cells patrolling the interstitial fluid are often centrifuged and discarded. Only in cases of extreme central nervous system inflammation, such as meningitis or encephalitis, are CSF cells utilized as a diagnostic. Blood cells, on the other hand, are routinely used to assess health and disease. The composition of blood and CSF cells are distinct (3), with $\mathrm{CD}^{+}$and $\mathrm{CD} 8^{+} \mathrm{T}$ cells, plasmacytoid dendritic cells, and CD56 ${ }^{\text {high }}$ natural killer (NK) cells enriched in the CSF, while monocytes, granulocytes, myeloid dendritic cells, basophils, and B cells are not as abundant (1). CSF cell density is also drastically lower with 1,000-3000 cells per $\mathrm{mL}$ versus millions per $\mathrm{mL}$ for blood (3-5). In comparison to blood, T lymphocytes are the most abundant cell type of the CSF and are comprised of more activated, antigen experienced cells.

To our knowledge, only the multiple sclerosis field has rigorously evaluated live CSF cells (3, 5-14), with sparse investigations in the context of AD $(15,16)$, narcolepsy (17), Rasmussen encephalitis (18), and paraneoplastic cerebellar degeneration (19). Lueg et al. report increased activation of $\mathrm{CD} 4^{+}$and $\mathrm{CD} 8^{+} \mathrm{T}$ cells in patients with $\mathrm{AD}$ and mild cognitive impairment (MCI) (16). Our group recently demonstrated the presence of clonally expanded, cytotoxic T cells in patients with AD and MCI (15). Similarly, Latorre et al. found hypocretinspecific $\mathrm{CD} 8^{+} \mathrm{T}$ cells in patients with narcolepsy (17). Cumulatively, these advances emphasize the relevance of CSF cells to the study of neurologic disease.

Most of the aforementioned studies have relied on freshy isolated CSF cells (3, 5, 6, 9-14, 16-20), or merely extraction of genomic DNA or RNA from frozen cells $(7,8)$. Analyzing fresh CSF cells provides the highest number of viable cells and the closest approximation of their endogenous physiology, but introduces batch effects and time restrictions. Conversely, cryopreservation allows for parallel analysis of multiple samples acquired longitudinally. Increased evidence for cellular changes within the CSF warrants a standardized protocol for the long-term preservation of CSF immune cells. Here we report a method for the long-term storage and subsequent analysis of CSF cells by scRNAseq. This method will enable researchers to interpret undiscovered neurodegenerative disease mechanisms.

\section{RESULTS}

We developed a standardized workflow for the isolation, cryopreservation and sequencing of CSF immune cells (Figure 1a). We first enumerated freshly isolated cells from $10 \mathrm{~mL}$ of CSF obtained by lumbar puncture from 18 subjects. Following centrifugation, we recovered an average of 15,121 ( $\pm 2,948$ s.e.m.) live cells per subject (Figure 1b). Prior to cryopreservation, cells had an average viability of $58 \%( \pm 6.8 \%$ s.e.m.; Figure 1c). After cryopreservation, cells were thawed at $37^{\circ} \mathrm{C}$ and live cells were sorted using a live/dead dye (Figure 1d). After thawing, an average of 4,961 ( \pm 506.1 s.e.m.) live cells were recovered by flow cytometry sorting (Figure $1 \mathrm{e})$ and $71 \%( \pm 2.0 \%$ s.e.m.) of scatter-gated cells were viable (Figure $1 \mathrm{f})$. While a considerable number of cells are lost with thawing, we did not detect an impact of the length of storage and cell viability $\left(\mathrm{R}^{2}=0.0079\right.$; Figure $1 \mathrm{~g})$.

After thawing and sorting live CSF cells, we obtained mRNA from an average of 1,323 cells $( \pm 106.1$ s.e.m.) per subject (Figure 2a). Clustering of scRNAseq data revealed $\mathrm{CD}^{+}$and $\mathrm{CD} 4^{+} \mathrm{T}$ cells, innate immune cells, natural killer cells, plasma cells, and B cells (Figure 2b). We detected genes expressed by plasma cells

(JCHAIN), NK cells (NKG7, GNLY), monocytes/macrophages (CD68, ITGAM and CD14), dendritic cells 
(ITGAX), and T cells (CD27, IL7R, CCR7, CD4, CD8B, and CD3D; Figure 2c). Importantly, we did not detect platelet genes, such as $P P B P$, confirming that our CSF samples were not contaminated with blood.

After clustering and annotating cells, we calculated the cumulative proportion of each cell type for all subjects (Figure 2d) and for individual subjects (Figure 2e). $\mathrm{CD}^{+} \mathrm{T}$ cells were the most abundant cell type (66\% $\pm 2.6 \%$ s.e.m.), followed by $\mathrm{CD}^{+} \mathrm{T}$ Cells ( $19 \% \pm 1.5 \%$ s.e.m.), innate immune cells (i.e. monocytes and dendritic cells; $8.5 \% \pm 2.6 \%$ s.e.m.), NK cells $(1.8 \% \pm 0.047 \%$ s.e.m), plasma cells $(0.49 \% \pm 0.15 \%$ s.e.m. $)$, and B cells $(0.31 \% \pm 1.7 \%$ s.e.m. $)$. We also detected a cluster comprised of both $\mathrm{CD}^{+} \mathrm{T}$ cells and $\mathrm{CD} 8^{+} \mathrm{T}$ cells $(4.8 \% \pm 1.7 \%$ s.e.m.) (Figure 2e). In summary, these results indicate that our method of long-term preservation of CSF cells is suitable for downstream applications such as scRNAseq.

\section{DISCUSSION}

Most studies performed on human cells rely on peripheral blood mononuclear cells. However, utilizing peripheral cells as a read-out of immune changes in neurodegeneration limits our ability to understand central immunity. CSF cells, on the other hand, provide a way to directly study immunology in the central nervous system. Indeed, recent studies of intrathecal immunity by us and others have shown this understudied immune compartment to be relevant to the pathobiology of neurodegenerative diseases $(3,5-20)$. Yet CSF cells have been widely understudied because of the invasive method of CSF extraction (typically lumbar puncture), and because cells are often discarded for proteomic analysis. Cells not discarded are typically analyzed fresh - typically by flow cytometry-which limits the scope of analysis.

Our simple and quick method allows for the preservation of an average 4,961 live cells after thawing, with no relationship between length of storage and cell viability. Importantly, the composition of cells from our method resembles that of freshly processed CSF cells reported in the literature. We find $66 \%$ of intrathecal cells are CD4 $4^{+}$ $\mathrm{T}$ cells and $19 \%$ are $\mathrm{CD} 8^{+} \mathrm{T}$ cells, which agrees with the $60-83 \%$ and $11-20 \%$ range reported by others, respectively $(3,21)$. We find that monocytes and dendritic cells combined make up $8.5 \%$ of CSF cells, which is similar to a previous study that found that monocytes make up 5-12\% and dendritic cells comprise less than $4 \%$ of intrathecal cells (3). Unfortunately, we were not able to determine the proportion of monocytes and dendritic cells separately, as they did not form distinct clusters in our sequencing analysis. We also detected small proportions of NK cells $(1.8 \%)$, plasma cells $(0.49 \%)$ and B cells $(0.31 \%)$. Han et al. report that natural killer cells make up 5\% - which is slightly higher than our observations - and others report that B cells typically make up less than $1 \%$ of CSF cells $(3,21)$. Altogether, while we lose minor proportions of NK and B cells, the composition of cells processed using our method of long-term preservation largely reflects that of freshly isolated CSF cells.

While studying fresh CSF cells provides maximal cell recovery, our method preserves major CSF cell types and their endogenous composition. Importantly, cryopreservation eliminates unavoidable batch effects and time constraints introduced from analyzing freshly isolated CSF cells. Therefore, cryopreservation of CSF cells allows for more complex and meaningful experiments on samples acquired longitudinally.

\section{CONCLUSIONS}

In conclusion, we provide a detailed method for long-term preservation of CSF cells and subsequent analysis by scRNAseq. We demonstrate that researchers can preserve CSF cells long-term and obtain valuable molecular information. However, we caution that freezing and thawing under this protocol may select for cells that better survive this process. Nonetheless, we anticipate that this simple protocol will enable other researchers 
to study CSF cells in a more robust and standardized way, providing important insight into intrathecal immunity in neurodegenerative diseases.

\section{METHODS}

\section{Study participants}

Samples were acquired through the Stanford Brain Rejuvenation Program, the NIA funded Stanford Alzheimer's Disease Research Center (ADRC), the University of California at San Francisco ADRC and the University of California at San Diego ADRC. Collection of CSF was approved by the Institutional Review Board of each university; written consent was obtained from all subjects. A total of 34 living subjects were used in this study. The 34 subjects included 10 subjects with Parkinson's disease (PD), 3 with Lewey-body dementia (LBD), 4 subjects with Alzheimer's disease (AD), 5 patients with Mild Cognitive Impairment (MCI), and 12 healthy controls.

\section{Cryopreservation of CSF cells}

CSF was collected by lumbar puncture, then centrifuged at $300 r c f$ for 10 minutes at $4{ }^{\circ} \mathrm{C}$ to pellet immune cells. Importantly, CSF samples were checked for blood contamination by examining the pellet for the presence of red blood cells by eye. An example of a CSF sample contaminated with blood is shown in Supplemental Figure 1a. Note that cells should remain at $4{ }^{\circ} \mathrm{C}$ until they are further processed, but it is best to freeze the cells as quickly as possible to limit cell death. The supernatant (cell free CSF) was aliquoted, carefully leaving behind $100 \mu \mathrm{l}$ of CSF with the pelleted cells. $100 \mu \mathrm{l}$ of CSF was left so that cells were concentrated enough for counting and viability measurements. The pelleted cells were then gently resuspended in the $100 \mu \mathrm{l} \mathrm{CSF}$ and $10 \mu \mathrm{l}$ of resuspended cells were then removed for counting. Importantly, cells were gently resuspended by first tapping the bottom of the tube and then gently triturating 10 times, making sure not to touch the pipette tip to the edge of the tube. Then $10 \mu \mathrm{CSF}$ was removed and mixed with $10 \mu$ trypan blue to assess red blood cell content and viability. Cells were then visualized on a TC20 automated cell counter (BioRad) and cell number, viability and the presence or absence of red blood cells was recorded. An example of a CSF sample contaminated with blood is shown in Supplemental Figure 1b. CSF samples contaminated with blood were discarded. The resuspended cells were then mixed with $900 \mu \mathrm{l}$ Recovery Cell Culture Freezing Medium (Thermo Fisher Scientific). This medium is an optimized version of the typical freezing medium, containing high-glucose Dulbecco's Modified Eagle Medium with $10 \%$ serum and $10 \%$ dimethyl sulfoxide. We utilized this medium because it is quality tested for $\mathrm{pH}$, osmolality, sterility, and endotoxin and each lot is quality tested on $\mathrm{CHO}-\mathrm{K} 1$ cells. The freezing medium was first thawed at $37{ }^{\circ} \mathrm{C}$, aliquoted, and stored at $-20{ }^{\circ} \mathrm{C}$. Before use, the medium was thawed at $37{ }^{\circ} \mathrm{C}$ and kept on ice. After each aliquot was thawed, the freezing medium was stored at $4{ }^{\circ} \mathrm{C}$ for up to one month. All samples were frozen overnight at $-80{ }^{\circ} \mathrm{C}$ in a Mr. Frosty freezing container (Thermo Fisher Scientific) and transferred the following day to liquid nitrogen for storage. CSF cells were stored in liquid nitrogen on average 266 days.

\section{Preparation of frozen CSF cells for analysis}

CSF cells were thawed at $37{ }^{\circ} \mathrm{C}$ in a water bath with the media submerged and the top of the tube out of the water. Cells were kept in the water bath for as little as possible and removed when the media was nearly completely thawed. The cells were then removed and gently pipetted into a $5 \mathrm{~mL}$ flow cytometry tube containing $3 \mathrm{~mL}$ prewarmed $\left(37^{\circ} \mathrm{C}\right)$ sorting buffer (PBS with $0.04 \%$ bovine serum albumin (BSA)). The tube was then rinsed once with the sorting buffer and placed into the same flow cytometry tube. Cells were then centrifuged at $350 \mathrm{rcf}$ for ten minutes. The supernatant was removed, and cells were resuspended in $500 \mu$ l sorting buffer. $1 / 2 \mu 1$ of Sytox Red (Thermo Fisher Scientific) was added to the sample immediately before sorting by flow cytometry. Live cells were sorted into $1.5 \mathrm{~mL}$ Eppendorf tubes containing $750 \mathrm{uL}$ sorting buffer. Once all the samples were sorted, cells were spun at $350 \mathrm{rcf}$ at $4{ }^{\circ} \mathrm{C}$ in a spinning bucket centrifuge for 7 minutes. The supernatant was then removed, 
leaving behind $10 \mu \mathrm{l} .10 \mu \mathrm{l}$ was left behind to resuspend the CSF cells and load the entire volume for droplet scRNA-seq.

\section{Drop-seq of CSF cells}

Chromium Single Cell 5' Library \& Gel Bead kit, Chromium Single Cell 5' Library construction kit, Chromium Single Cell A Chip Kit, and Chromium i7 Multiplex kit (10X Genomics) were used for scRNAseq of CSF cells. We followed 10x Genomic's User Guide for library construction. The only change we made to their protocol was in Step 1, GEM Generation \& Barcoding. The user guide recommends loading a certain volume of cell suspension stock depending on the concentration of the cell suspension and the user's desired cell recovery. However, because CSF contains such low cell numbers, we loaded all the cells that were resuspended in $10 \mu$ of sorting buffer. We then added the $10 \mu \mathrm{l}$ of cell suspension and $21.7 \mu \mathrm{l}$ of nuclease free water, which results in the same total volume of cell suspension/water that the user guide recommends.

After library construction, libraries were sequenced by Novogene on a Novoseq S4 sequencer and FASTQ files were generated by Novogene. Cell Ranger v.3.0.2 was used to generate gene-expression matrices for CSF cells. Reads from the 10X v.2 5' paired library were mapped to the human genome build GRCh38 3.0.0. The 5' geneexpression libraries were then analyzed with the Cell Ranger count pipeline and the resulting expression matrix was used for further analysis in the Seurat package v.3.0.

\section{Clustering of CSF cells}

Individual sample expression matrices were loaded into $\mathrm{R}$ using the function Read10x under the Matrix package v.1.2-15. The expression matrix for each sample was merged into one Seurat object using the CreateSeuratObject and MergeSeurat functions. The Seurat package v.3.0 was used for filtering, variable gene selection, normalization, scaling, dimensionality reduction, clustering and visualization. Genes were excluded if they were expressed in fewer than 10 cells and cells were excluded if they expressed fewer than 200 genes. Cells that expressed more than 1,800 genes, more than 7,500 UMIs and more than 10\% mitochondrial genes were excluded from the analysis. The sctransform normalization method was used to normalize, scale, select variable genes and regress out sequencing and experimental batch, mitochondrial mapping percentage and the number of UMIs. After filtering and normalization, there were 26,797 cells and 14,953 genes. Following PCA, 11 principle components were selected for clustering. A resolution of 0.8 was used with t-SNE visualization.

\section{LIST OF ABBREVIATIONS}

CSF = cerebrospinal fluid; scRNAseq = single cell RNA sequencing; Alzheimer's disease = AD; Mild cognitive impairment $=\mathrm{MCI}$; Natural killer $(\mathrm{NK})$

\section{DECLARATIONS}

\section{Ethics approval and consent to participate}

Collection of CSF was approved by the Institutional Review Board of each university, and written consent was obtained from all subjects

\section{Consent for publication}

Not applicable

\section{Availability of data and materials}


The scRNAseq datasets analyzed during the current study are available in the Gene Expression Omnibus (GEO) repository under accession number GSE134578. The fresh cell counts and flow cytometry data generated in the current study are available from the corresponding author upon request.

\section{Competing interests}

The authors declare that they have no competing interests.

\section{Funding}

This work was supported by a Glenn/American Federation for Aging Research (AFAR) Postdoctoral Fellowship for the Biology of Aging (D.G.), a National Institutes of Health National Institute on Aging (NIA) F32 Fellowship (AG055255-01A1) (D.G.), an Irene Diamond Fund/AFAR Postdoctoral Transition Award in Aging (D.G.) and the NIA funded Stanford Alzheimer's Disease Research Center P50AG047366.

\section{Author's contributions}

D.G. and O.L. designed experiments. D.G. and O.L. prepared the manuscript. D.G. conducted flow cytometry analysis and sorting. O.L. analyzed scRNAseq data. All authors edited, read and approved the final manuscript.

\section{Acknowledgements}

We thank V. Henderson and the entire Stanford Alzheimer's disease Reserach Center team; G. Kerchner and S. Sha for CSF collection and D. Channappa for CSF processing. 


\section{REFERENCES}

1. Perrin RJ, Fagan AM, Holtzman DM. Multimodal techniques for diagnosis and prognosis of Alzheimer's disease. Nature. 2009;461(7266):916-22.

2. Blennow K, Zetterberg H. The Past and the Future of Alzheimer's Disease Fluid Biomarkers. J Alzheimers Dis. 2018;62(3):1125-40.

3. Han S, Lin YC, Wu T, Salgado AD, Mexhitaj I, Wuest SC, et al. Comprehensive immunophenotyping of cerebrospinal fluid cells in patients with neuroimmunological diseases. J Immunol. 2014;192(6):2551-63.

4. Ransohoff RM, Engelhardt B. The anatomical and cellular basis of immune surveillance in the central nervous system. Nat Rev Immunol. 2012;12(9):623-35.

5. Schafflick D, Xu CA, Hartlehnert M, Cole M, Schulte-Mecklenbeck A, Lautwein T, et al. Integrated single cell analysis of blood and cerebrospinal fluid leukocytes in multiple sclerosis. Nat Commun. 2020;11(1):247.

6. Aly L, Yousef S, Schippling S, Jelcic I, Breiden P, Matschke J, et al. Central role of JC virus-specific CD4+ lymphocytes in progressive multi-focal leucoencephalopathy-immune reconstitution inflammatory syndrome. Brain. 2011;134(Pt 9):2687-702.

7. Lossius A, Johansen JN, Vartdal F, Robins H, Jurate Saltyte B, Holmoy T, et al. High-throughput sequencing of TCR repertoires in multiple sclerosis reveals intrathecal enrichment of EBV-reactive CD8+ $\mathrm{T}$ cells. Eur $\mathrm{J}$ Immunol. 2014;44(11):3439-52.

8. Salou M, Garcia A, Michel L, Gainche-Salmon A, Loussouarn D, Nicol B, et al. Expanded CD8 T-cell sharing between periphery and CNS in multiple sclerosis. Ann Clin Transl Neurol. 2015;2(6):609-22.

9. Johansen JN, Vartdal F, Desmarais C, Tutturen AE, de Souza GA, Lossius A, et al. Intrathecal BCR transcriptome in multiple sclerosis versus other neuroinflammation: Equally diverse and compartmentalized, but more mutated, biased and overlapping with the proteome. Clin Immunol. 2015;160(2):211-25.

10. Planas R, Metz I, Ortiz Y, Vilarrasa N, Jelcic I, Salinas-Riester G, et al. Central role of Th2/Tc2 lymphocytes in pattern II multiple sclerosis lesions. Ann Clin Transl Neurol. 2015;2(9):875-93.

11. Hoglund RA, Lossius A, Johansen JN, Homan J, Benth JS, Robins H, et al. In Silico Prediction Analysis of IdiotopeDriven T-B Cell Collaboration in Multiple Sclerosis. Front Immunol. 2017;8:1255.

12. Rathbone E, Durant L, Kinsella J, Parker AR, Hassan-Smith G, Douglas MR, et al. Cerebrospinal fluid immunoglobulin light chain ratios predict disease progression in multiple sclerosis. J Neurol Neurosurg Psychiatry. 2018;89(10):10449.

13. Beltran E, Gerdes LA, Hansen J, Flierl-Hecht A, Krebs S, Blum H, et al. Early adaptive immune activation detected in monozygotic twins with prodromal multiple sclerosis. J Clin Invest. 2019;129(11):4758-68.

14. Tomescu-Baciu A, Johansen JN, Holmoy T, Greiff V, Stensland M, de Souza GA, et al. Persistence of intrathecal oligoclonal B cells and IgG in multiple sclerosis. J Neuroimmunol. 2019;333:576966.

15. Gate D, Saligrama N, Leventhal O, Yang AC, Unger MS, Middeldorp J, et al. Clonally expanded CD8 T cells patrol the cerebrospinal fluid in Alzheimer's disease. Nature. 2020;577(7790):399-404.

16. Lueg G, Gross CC, Lohmann H, Johnen A, Kemmling A, Deppe M, et al. Clinical relevance of specific T-cell activation in the blood and cerebrospinal fluid of patients with mild Alzheimer's disease. Neurobiol Aging. 2015;36(1):81-9.

17. Latorre D, Kallweit U, Armentani E, Foglierini M, Mele F, Cassotta A, et al. T cells in patients with narcolepsy target self-antigens of hypocretin neurons. Nature. 2018;562(7725):63-8.

18. Schneider-Hohendorf T, Mohan H, Bien CG, Breuer J, Becker A, Gorlich D, et al. CD8(+) T-cell pathogenicity in Rasmussen encephalitis elucidated by large-scale T-cell receptor sequencing. Nat Commun. 2016;7:11153.

19. Albert ML, Austin LM, Darnell RB. Detection and treatment of activated T cells in the cerebrospinal fluid of patients with paraneoplastic cerebellar degeneration. Ann Neurol. 2000;47(1):9-17.

20. Hummert MW, Alvermann S, Gingele S, Gross CC, Wiendl H, Mirenska A, et al. Immunophenotyping of cerebrospinal fluid cells by Chipcytometry. J Neuroinflammation. 2018;15(1):160.

21. Kivisakk P, Mahad DJ, Callahan MK, Trebst C, Tucky B, Wei T, et al. Human cerebrospinal fluid central memory CD4+ T cells: evidence for trafficking through choroid plexus and meninges via P-selectin. Proc Natl Acad Sci U S A. 2003;100(14):8389-94. 


\section{FIGURE LEGENDS}

\section{Figure 1. Cryopreservation and analysis of thawed CSF immune cells}

a) Work flow for preserving CSF cells for scRNA-seq. b) Quantification of total live cells of freshly isolated CSF cells. Mean \pm s.e.m. c) Quantification of cell viability of freshly isolated CSF cells. Mean \pm s.e.m. d) Gating strategy for sorting live cryopreserved cells by flow cytometry. e) Quantification of live cell count (live singlets) from flow cytometry sorting. Mean \pm s.e.m. f) Quantification of viability (percent of live singlets out of all singlets) from sorting by flow cytometry. Mean \pm s.e.m. g) Linear regression showing no relationship between length of storage of cell viability with $r$ and $\mathrm{R}^{2}$ shown.

\section{Figure 2. Single cell RNA sequencing of CSF cells}

a) Quantification of the number of CSF cells captured for sequencing. b) CSF cells analyzed by drop-seq and clustered by multidimensional reduction with t-SNE, showing populations of immune cells. Data were pooled from three independent experiments. c) Dot plotting showing the number of cells expressing important markers genes (size of dot) and the level of expression (color of dot). Genes shown were those used to determine cell type of clusters. d) Quantification of the percentage of each cell type within the CSF (combining all subjects). e) Quantification of the percentage of each cell type within the CSF for each subject. Mean \pm s.e.m.

\section{Supplementary Figure 1. Identification of blood contamination in CSF}

a) Representative CSF pellet with visible blood contamination. b) Representative image from the automated cell counter showing CSF contaminated with red blood cells. Blue arrow head is a dead cell, green arrow head is a live cell, and red arrow head is a red blood cell. Cells with evidence of red blood cells were excluded from further analysis. 
Figure 1

a

1 Isolate CSF by lumbar puncture

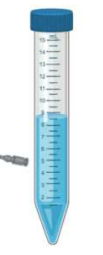

4
2

Pellet CSF cells by centrifugation

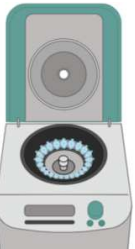

Thaw and sort live cells

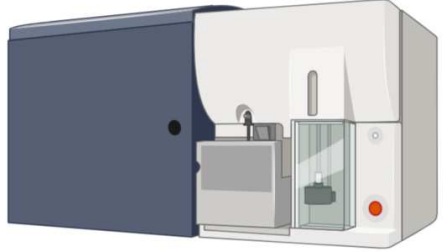

b

3

Cryopreserve cells

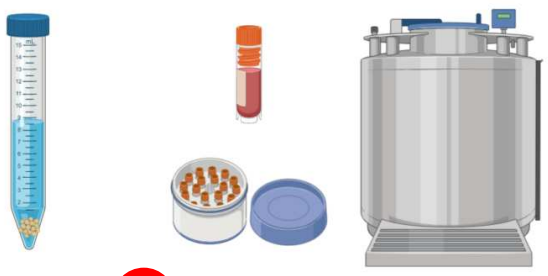

5 Barcode cells and RNA

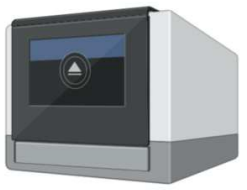

Fresh CSF live cell count

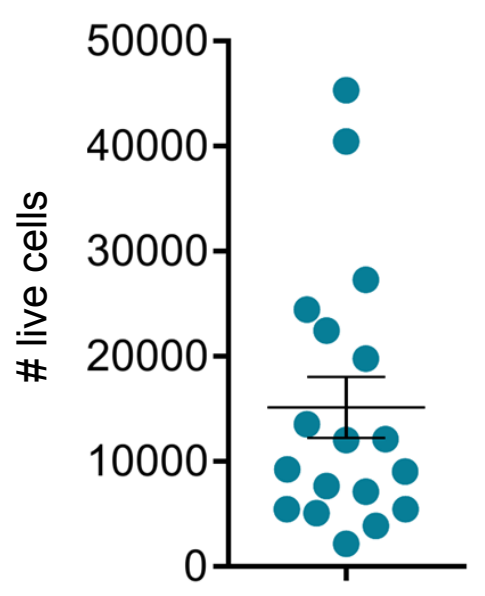

e Thawed live singlets

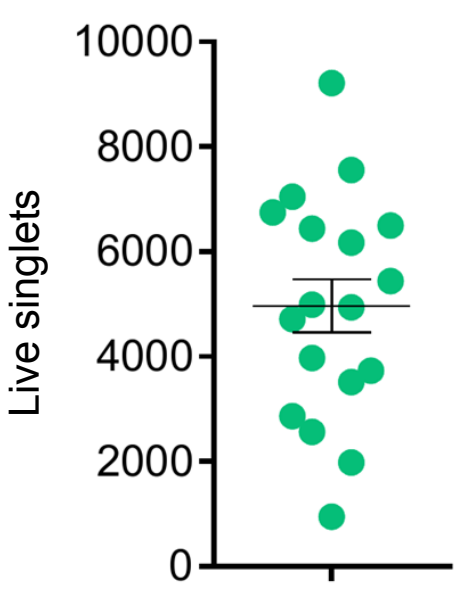

C

Fresh CSF cell viability

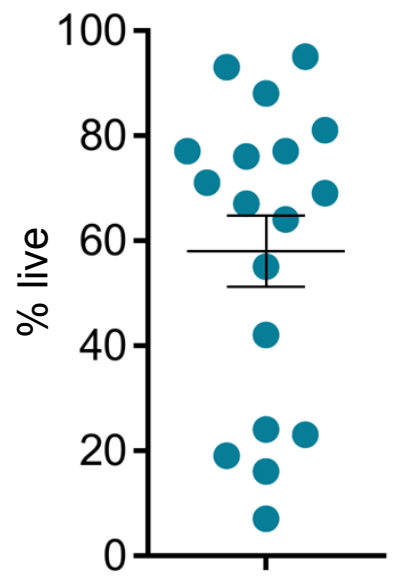

f

Thawed viability

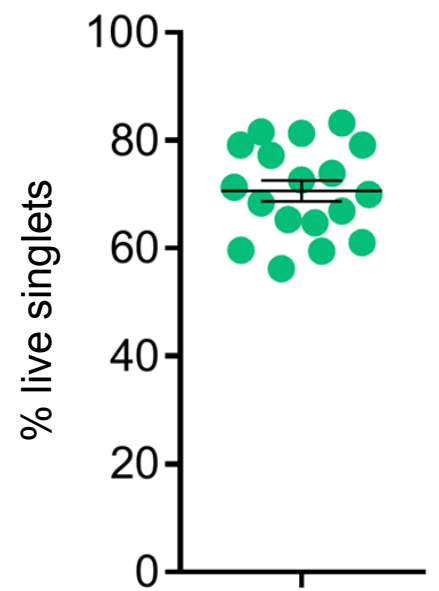

Association between live singlets and storage duration

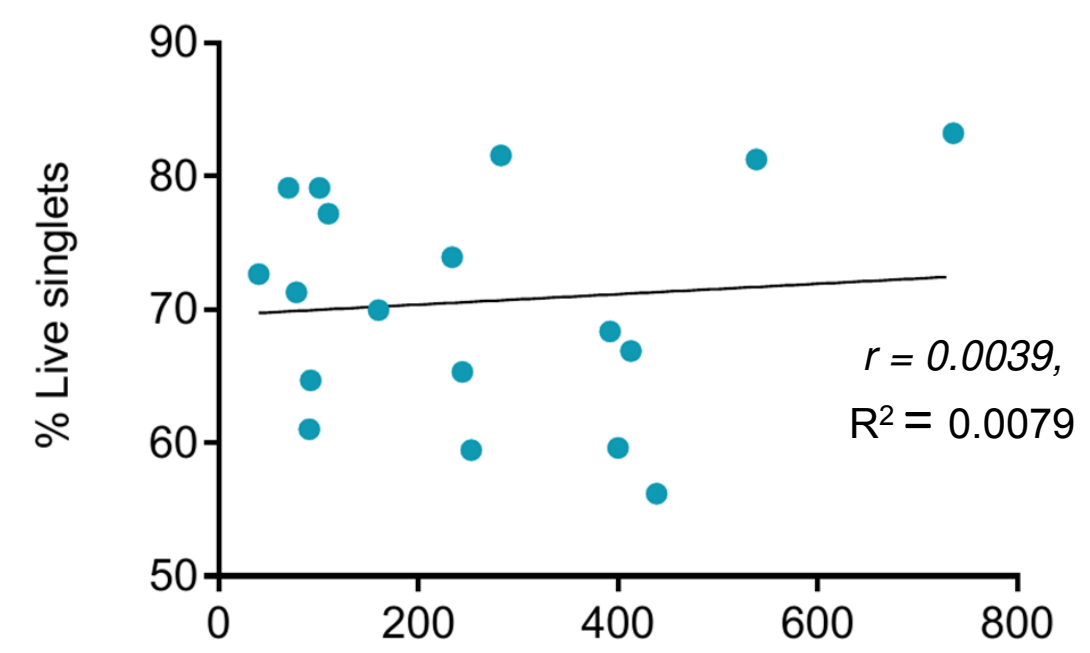

Length of Storage (days) 


\section{Supplementary Figure 1}
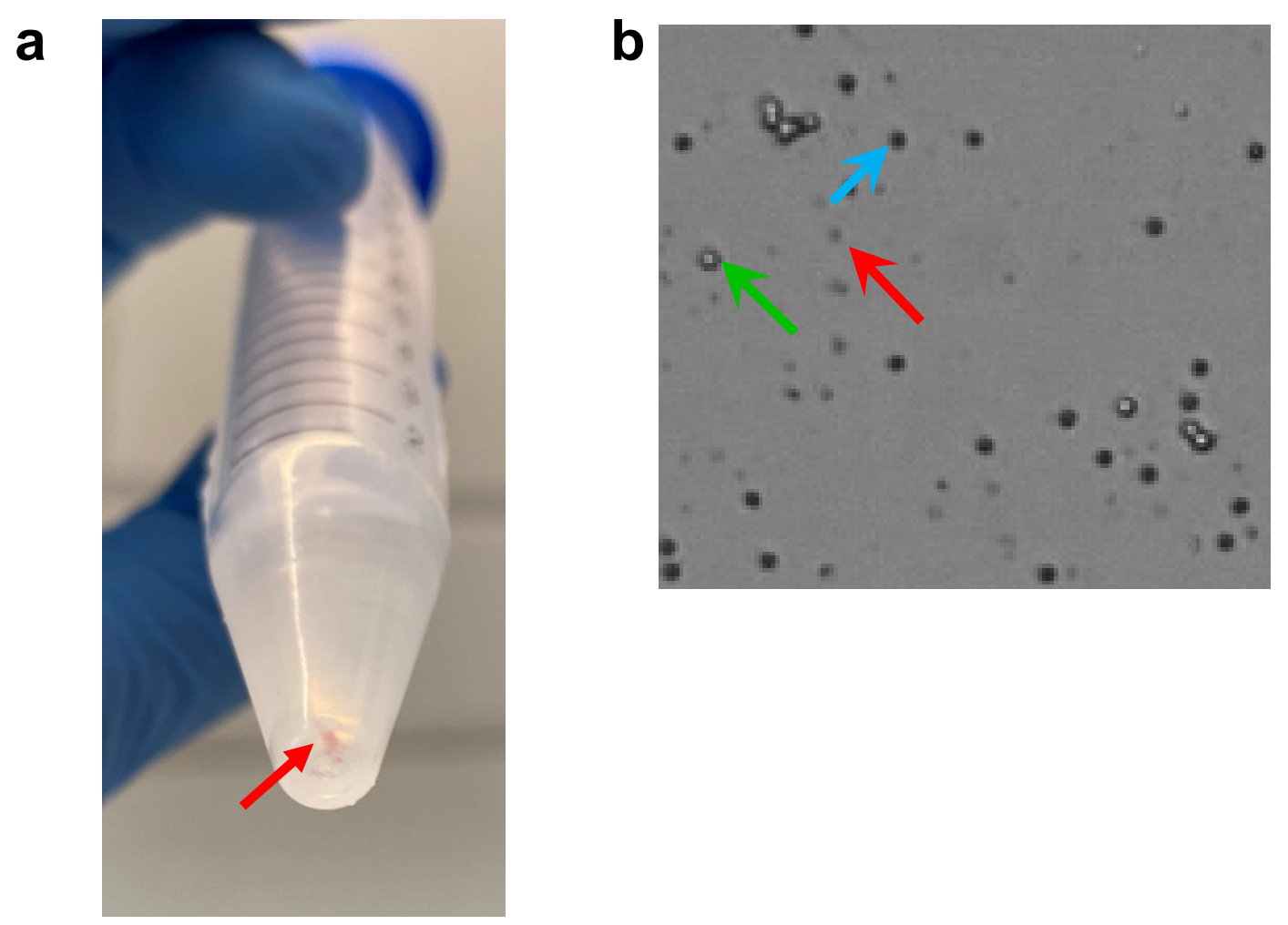


\section{Figures}

a

(1) Isolate CSF by lumbar puncture

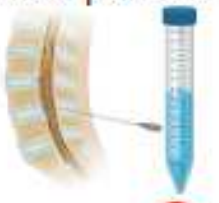

(4)

Thaw and sort live cells

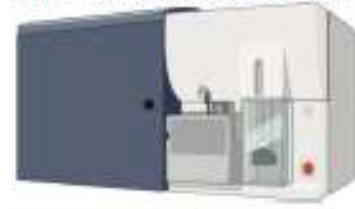

Pellet CSF cells

by centrifugation

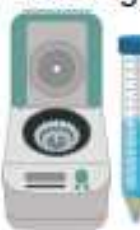

5

Barcode cells and RNA

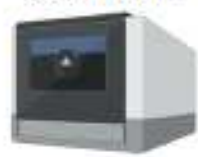

(3) Cryopreserve cells

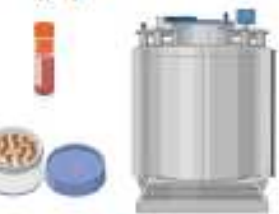

6 Sequence

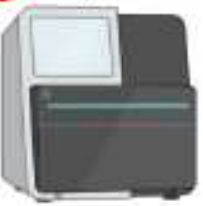

d

Gating Strategy for cryopreserved CSF cells

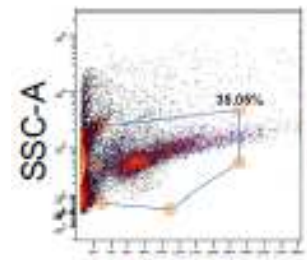

FSC-A

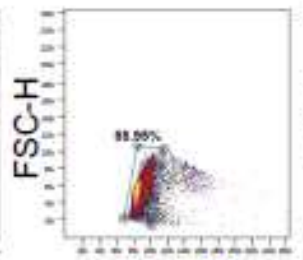

FSC-W

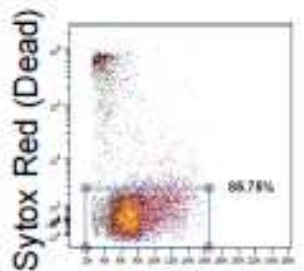

FSC-A

g

Association between live singlets and storage duration b

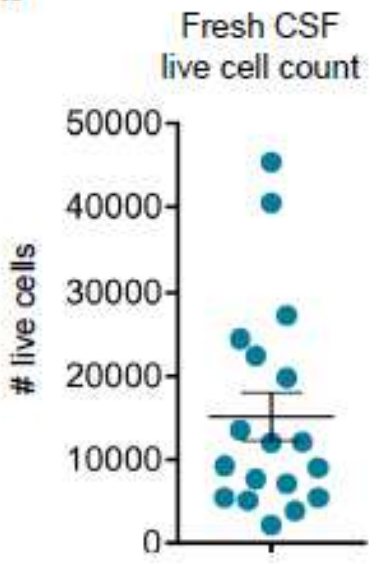

Fresh CSF

C

Figure 1
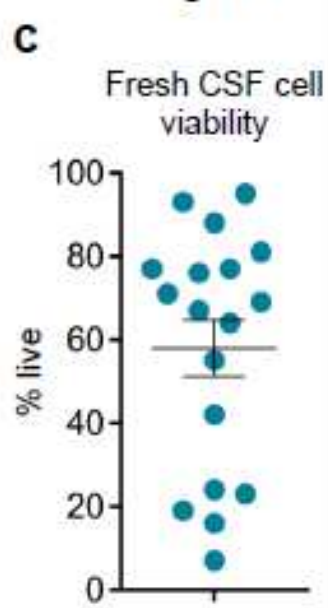

e

Thawed live singlets

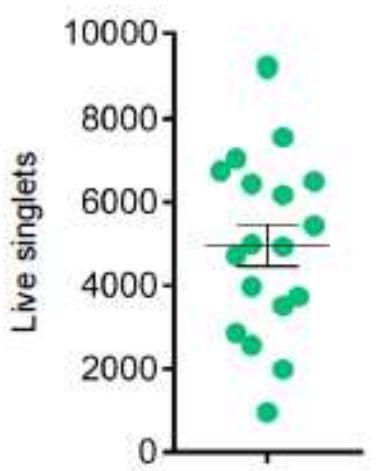

f

Thawed viability

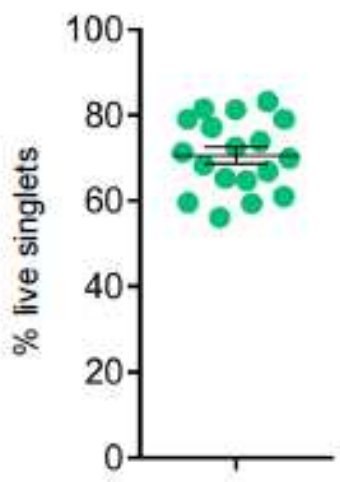

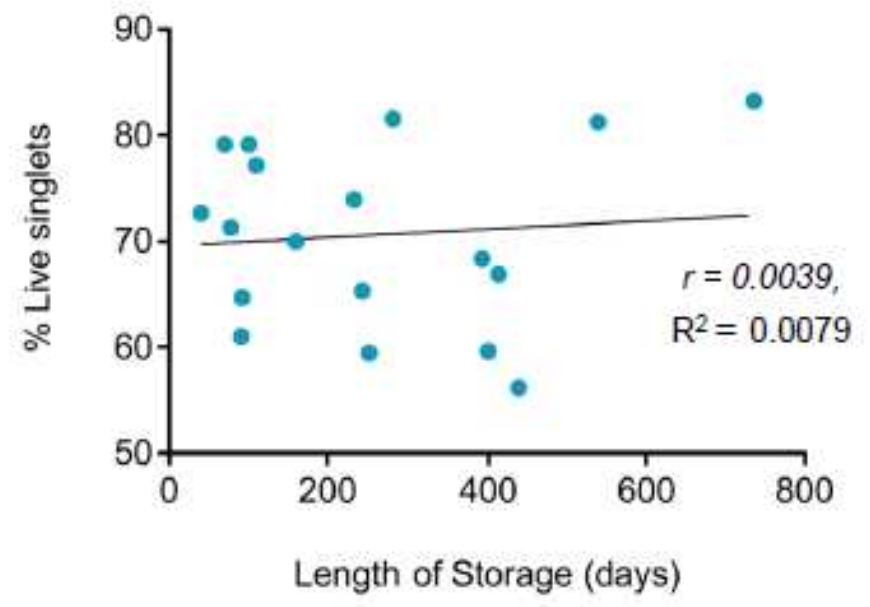

Figure 1

Cryopreservation and analysis of thawed CSF immune cells. a) Work flow for preserving CSF cells for scRNA-seq. b) Quantification of total live cells of freshly isolated CSF cells. Mean \pm s.e.m. c) Quantification of cell viability of freshly isolated CSF cells. Mean \pm s.e.m. d) Gating strategy for sorting 
live cryopreserved cells by flow cytometry. e) Quantification of live cell count (live singlets) from flow cytometry sorting. Mean \pm s.e.m. f) Quantification of viability (percent of live singlets out of all singlets) from sorting by flow cytometry. Mean \pm s.e.m. g) Linear regression showing no relationship between length of storage of cell viability with $r$ and R2 shown.
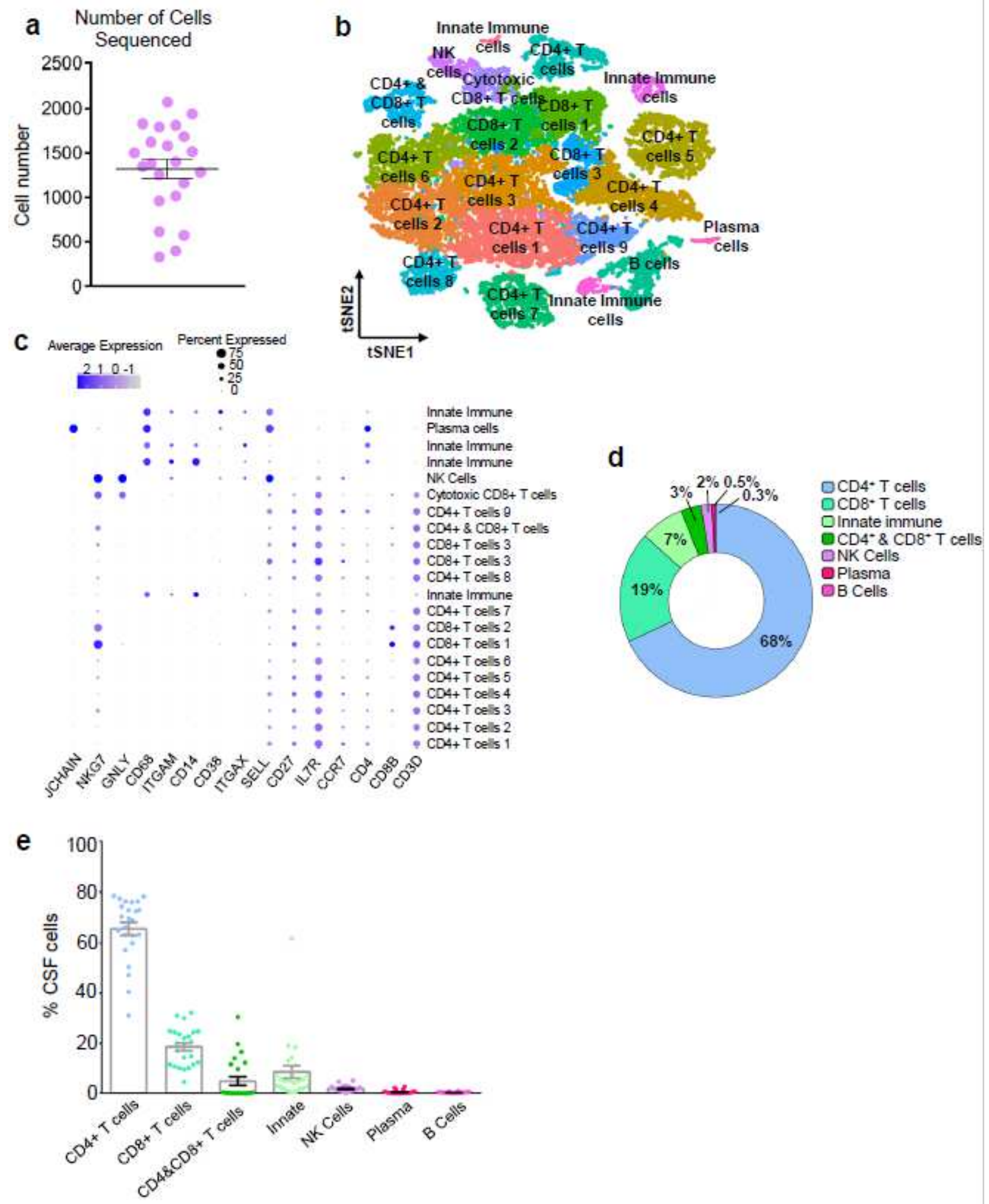

Figure 2 
Single cell RNA sequencing of CSF cells. a) Quantification of the number of CSF cells captured for sequencing. b) CSF cells analyzed by drop-seq and clustered by multidimensional reduction with t-SNE, showing populations of immune cells. Data were pooled from three independent experiments. c) Dot plotting showing the number of cells expressing important markers genes (size of dot) and the level of expression (color of dot). Genes shown were those used to determine cell type of clusters. $d$ ) Quantification of the percentage of each cell type within the CSF (combining all subjects). e) Quantification of the percentage of each cell type within the CSF for each subject. Mean \pm s.e.m.

\section{Supplementary Files}

This is a list of supplementary files associated with this preprint. Click to download.

- suppfig1.png 